\title{
Health Maintenance for Adult Patients with Inflammatory Bowel Disease
}

\author{
Jana G. Hashash, MD, MSc ${ }^{1, *}$ \\ Michael F. Picco, MD, PhD \\ Francis A. Farraye, MD, MSC ${ }^{1}$
}

\author{
Address \\ *,1 Inflammatory Bowel Disease Center, Division of Gastroenterology, Mayo Clinic, \\ Jacksonville, FL, USA \\ Email:AlHashash.Jana@mayo.edu \\ Published online: 22 November 2021 \\ ๑ The Author(s), under exclusive licence to Springer Science+Business Media, LLC, part of Springer Nature 2021
}

This article is part of the Topical Collection on Inflammatory Bowel Disease

Keywords Preventive care - Vaccinations - Healthcare maintenance - Screening · Inflammatory bowel disease

\begin{abstract}
Purpose of review This review serves as a summary of healthcare maintenance items that should be addressed when managing patients with inflammatory bowel disease (IBD). This manuscript discusses vaccine-preventable illnesses, cancer prevention recommendations, and other screenings that are important to gastroenterologists and primary care physicians caring for patients with IBD.

Recent findings Patients with IBD often require immunomodulator agents and/or biologics to induce and maintain disease remission which can increase the risk of developing several infections. Also, subsets of patients with IBD are at an increased risk for a number of malignancies including colon, cervical, and skin cancers.

Summary Staying up-to-date with health care maintenance of patients with IBD is critical, especially given their increased risk for vaccine-preventable infections as well as comorbidities such as cancers, bone health, and mood disorders. Gastroenterologists and primary care physicians should familiarize themselves with the required screenings and vaccines that are recommended for adult patients with IBD, particularly those who are immunosuppressed.
\end{abstract}




\section{Introduction}

Health maintenance is crucial for patients with inflammatory bowel diseases (IBD), especially those who are receiving immunosuppressive therapy or those with plans to be started on such drugs. IBD is a chronic condition that can affect patients of all ages. It does not only involve the gastrointestinal tract but may impact the entire body. Up to $40 \%$ of patients with IBD have associated extra-intestinal manifestations which may involve the eyes, skin, joints, and liver [1]. The importance of healthcare maintenance does not only include vaccinations, but also involves screening and surveillance for certain cancers, depression, osteoporosis, and smoking, among other conditions. Many patients with IBD are on immunosuppressive medications such as corticosteroids, thiopurines, methotrexate, small molecules, and/or biologic agents making them not only at an increased risk for the development of opportunistic infections and malignancies, but for developing a more severe and complicated course [2].

Patients with IBD often consider their treating gastroenterologist as their primary care provider. Previous studies have shown that rates of preventative care for patients with IBD are lower than that of the general population $[2,3]$. This is likely due to several reasons. Primary care providers may be less comfortable making recommendations about immunizations particularly in the setting of immunosuppression due to a lack of knowledge regarding required vaccinations and screenings for patients with IBD. Gastroenterologists, being more focused on the gastrointestinal tract and not addressing healthcare maintenance issues due to time constraints, and/or their inability to provide vaccinations in their office due to extra cost and storage issues, may defer this responsibility to primary care physicians $[4,5]$. For this reason, it is imperative that gastroenterologists work closely with primary care clinicians as one team in order to make sure that their patients with IBD are receiving appropriate and up-to-date healthcare maintenance $[6,7]$. Treating gastroenterologists should make recommendations about vaccines, communicate these recommendations to primary physicians, and define who is responsible for vaccine administration, health maintenance, and screenings [ $\left[\mathbf{\bullet}^{\bullet}\right.$ ].It is also of paramount importance for gastroenterologists to be able to recognize the other primary, secondary, and tertiary prevention tasks required for every patient with IBD [9]. Several checklists have been created to facilitate healthcare maintenance for patients with $\operatorname{IBD}[10 \bullet, 11 \bullet]$.

In this manuscript, we will discuss the different healthcare maintenance aspects that need to be addressed by clinicians caring for patients with IBD. This paper will address vaccine-preventable illnesses, screening for malignancy, osteoporosis, and mental health.

Vaccine-preventable illnesses

Vaccines are important for the prevention of a number of infectious illnesses $[12 \bullet \bullet$.There has been no association between vaccination causing flares of underlying IBD $[13,14]$. Among patients with IBD, it is very important to make sure that patients have received their age-appropriate vaccines ideally prior to initiation of immunosuppressive medications for optimal immune response [15]. Equally as important, family members of patients with IBD should also be up-to-date with their immunizations.

Non-live (inactive/killed) vaccines can be administered to all patients, regardless of their immunosuppression status per the Centers for Disease Control (CDC) recommendation, Advisory Committee on Immunization Practices (ACIP), and the Infectious Disease Society of America (IDSA) [16-19]. These vaccines include the inactivated influenza vaccine, pneumococcal vaccine (PCV13 and PPSV23), tetanus, diphtheria, and pertussis (Tdap), meningococcal vaccine, hepatitis $\mathrm{A}$ and $\mathrm{B}$ vaccines, human papillomavirus $(\mathrm{HPV})$, and the inactivated recombinant herpes zoster vaccine [ $8 \bullet \bullet$.Live 
vaccines, on the contrary, are contraindicated in immunosuppressed patients. Per the CDC, patients with IBD are considered severely immunocompromised if on (1) prednisone $\geq 20 \mathrm{mg}$ daily for at least 2 weeks, (2) thiopurines ( $\geq 1.5$ $\mathrm{mg} / \mathrm{kg}$ of 6 -mercaptopurine or $\geq 3 \mathrm{mg} / \mathrm{kg}$ of azathioprine), or (3) methotrexate $(\geq 0.4 \mathrm{mg} / \mathrm{kg}$ weekly) [20]. Additionally, patients on biologic agents are considered immunosuppressed. Novel messenger RNA (mRNA) and adenovirus COVID-19 vaccines are approved and are recommended in all patients with IBD. Given the high likelihood of needing immunosuppressive medication during their illness, required vaccinations should be given to all patients with IBD whenever possible. It is very important for gastroenterologists to vaccinate their patients with IBD early on prior to initiation of immunosuppression to achieve a maximum immune response [21]. Table 1 summarizes the immunization schedule of adult patients with IBD.

This review will not address vaccinations of patients with IBD planning international travel. The reader is referred to the CDC website as well as the ACG preventive care clinical guideline $[8 \bullet \bullet, 20,22]$.

Influenza vaccine

Patients with IBD have been shown to have a higher risk for contracting influenza compared to individuals with no IBD. Steroid use was found to be the only medication to independently be associated with this risk [23]. Additionally, patients with IBD were found to have a higher likelihood for hospitalization. Studies have demonstrated that patients with IBD receiving immunosuppressive therapies are at an increased risk for developing influenza and if infected, they were found to have worse outcomes defined as higher rates of hospitalization and superimposed pneumonia compared to individuals without IBD [24]. Consequently, all patients with IBD should receive the inactivated non-live influenza vaccine annually, regardless of their immunosuppression status. Additionally, household members of patients with IBD should receive the annual influenza vaccine also. Timing of influenza vaccine administration should not be delayed based on the timing of biologic agent dose administration. Despite the blunted immune response noted among immunosuppressed patients with IBD, the vaccine still provides some protection [25-28]. In one study, up to $80 \%$ of patients with IBD on infliximab who received the influenza vaccine mounted a serologic response [29].

Currently, the most commonly administered influenza vaccines are the standard dose and the high dose preparations of the inactivated influenza vaccines. It has been shown that the high-dose inactivated influenza vaccine may lead to higher antibodies in patients with IBD who are receiving anti-tumor necrosis factor (anti-TNF) monotherapy when compared to the standard dose administration therefore this is what has been recommended in a recent expert review [30, 31]. The live attenuated influenza vaccine is contraindicated among patients with IBD who are receiving immunosuppressive medications. 


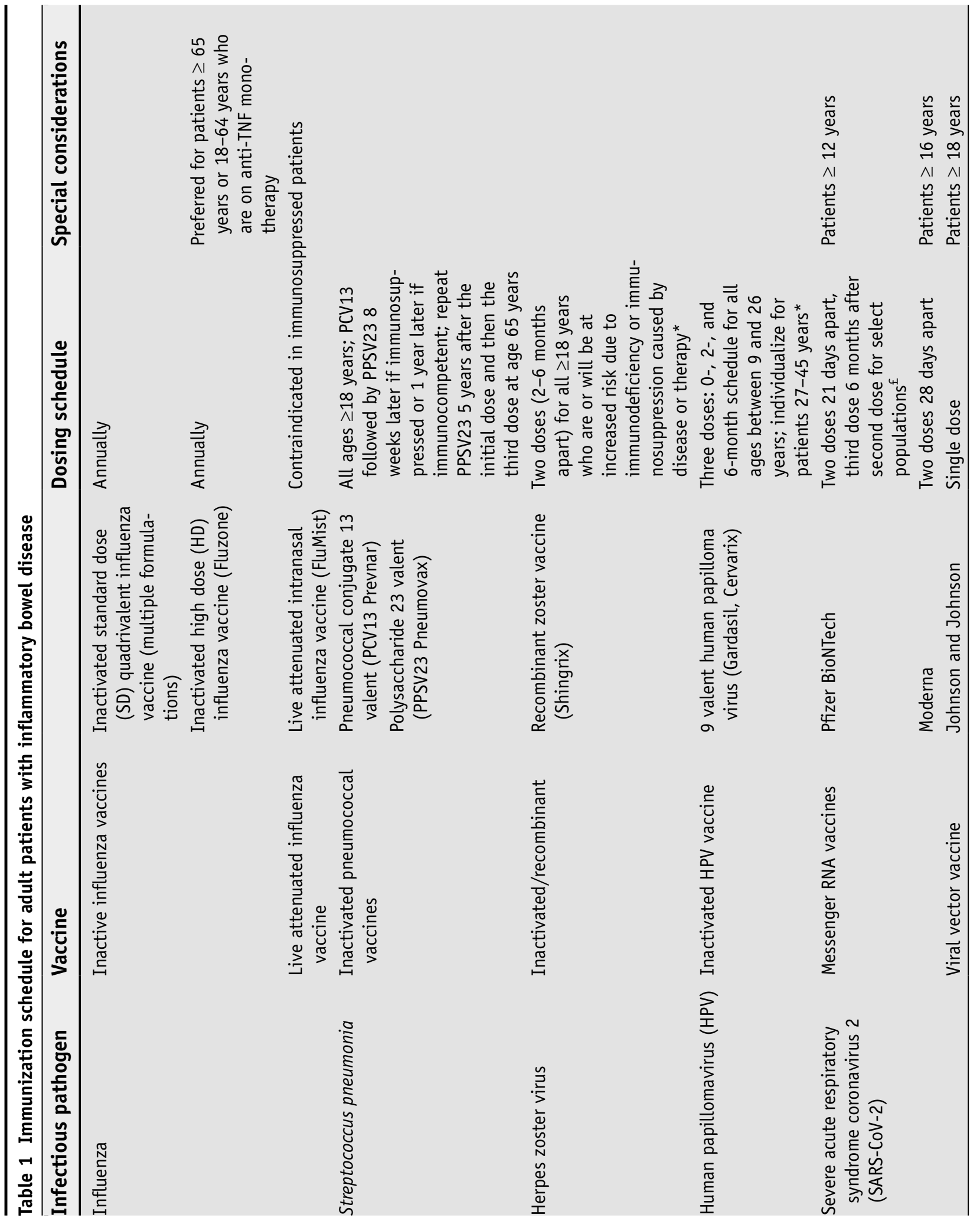




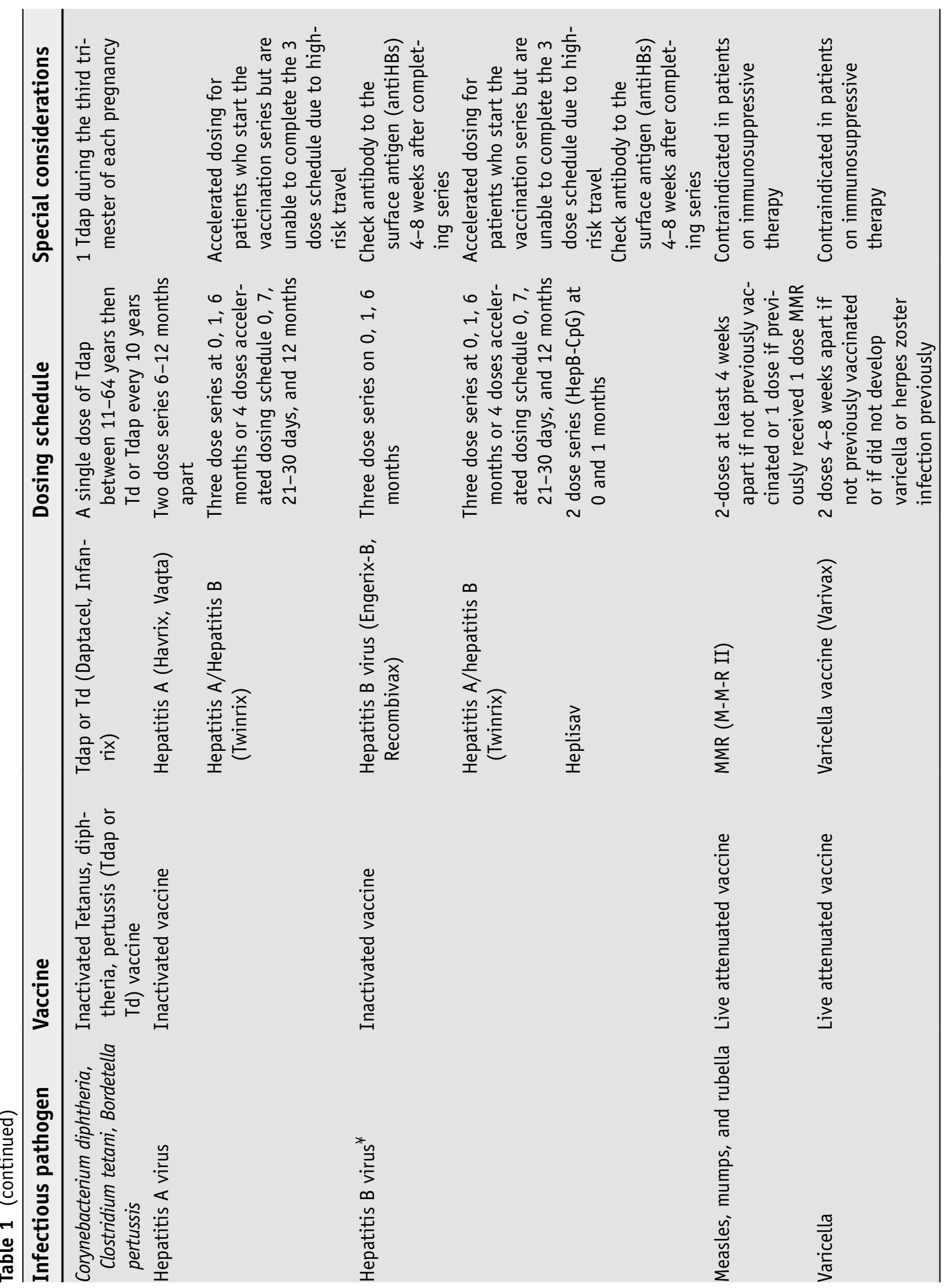




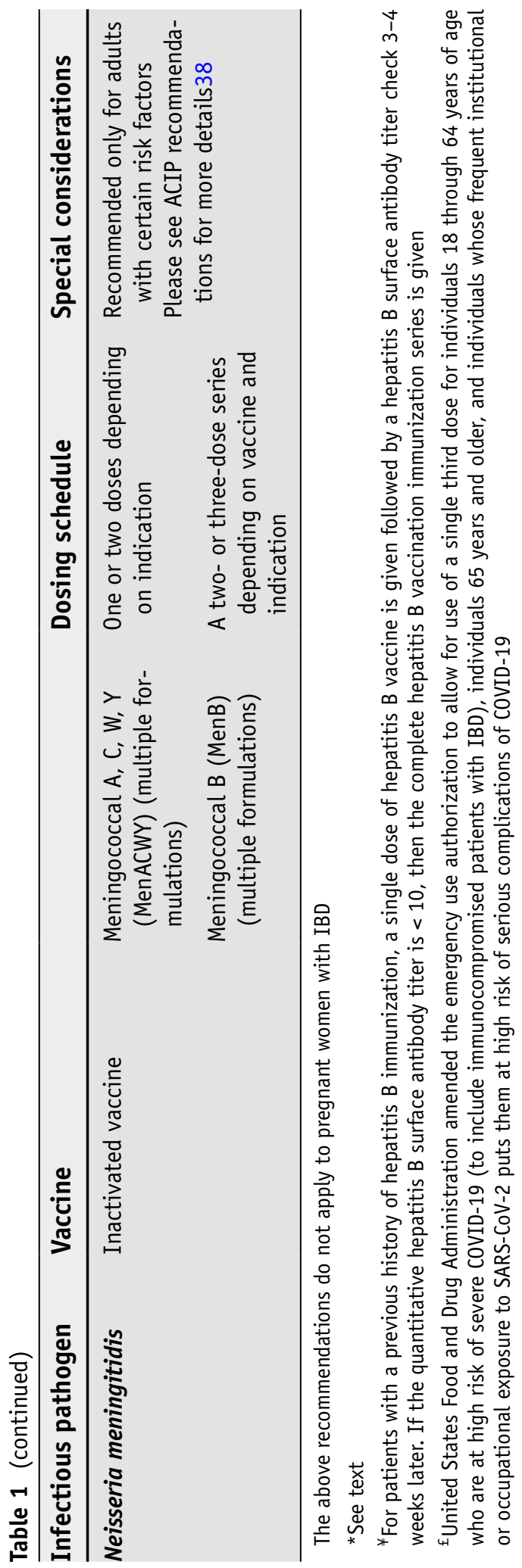


Pneumococcal vaccines

Patients with IBD are at an increased risk for developing pneumococcal pneumonia compared to the general population [32]. Although there are some studies showing an increased risk for pneumococcal pneumonia in patients receiving immunosuppression, a nationwide Danish cohort study showed that immunosuppressive medications, namely steroids and anti-TNF agents, did not increase the risk of invasive pneumococcal disease among patients with IBD $[32,33]$. Currently, there are 2 pneumococcal vaccines: the pneumococcal conjugate vaccine PCV-13 (Prevnar $13^{\circledR}$ ) and the pneumococcal polysaccharide PPSV23 vaccine (Pneumovax ${ }^{\circledR} 23$, and both can be given to patients with IBD regardless of immunosuppression status.

For immunocompromised patients, PCV-13 is given first followed by PPSV23, 8 weeks later. In immunocompetent patients, PPSV23 should be given 1 year after PCV-13. Additional PPSV23 vaccine doses should be given 5 years after the initial PPSV23 dose and then dose 3 at the age of 65 years. In the event that an immunocompromised patient is given PPSV23 first, it is recommended that patients receive PCV-13 1 year later. As with all vaccines, patients should be vaccinated early on after diagnosis of IBD and preferably before initiation of immunosuppression as to not have a blunted immune vaccine response. However, in patients already on biologic agents, vaccination should not be delayed based on the timing of biologic treatment [34].

Herpes zoster vaccine

Patients with IBD are at increased risk for the development of herpes zoster compared to the general population,32]. and those receiving immunosuppression, especially tofacitinib, are at a further increased risk [35]. A recombinant zoster vaccine (Shingrix) was approved in 2017 as an alternative to the live zoster vaccine (Zostavax) which is no longer available in the USA. All individuals over the age of 50 years should receive the recombinant zoster vaccine. Experts have proposed that a subgroup of patients with IBD who are at high risk of developing herpes zoster should receive the vaccine earlier than 50 years and these include (1) 40 years and older with a history of herpes zoster, (2) repeated courses of steroids, (3) combination therapy and receiving steroids, (4) tofacitinib use with one of the following: oral corticosteroids at baseline, Asian race, history of diabetes mellitus, prior anti-TNF failure, or chronic tofacitinib use of $10 \mathrm{mg}$ twice a day $[30,35,36]$. In July 2021 , the FDA approved Shingrix for the prevention of herpes zoster in adults 18 years of age and older who are, or will be, at an increased risk of shingles because of immunodeficiency or immunosuppression. 
HPV vaccine

Patients with IBD who are receiving immunosuppression are at a 30\% increased risk for cervical neoplasia compared to the general population [37]. Persistent HPV infection and an immunocompromised system predispose to cervical cancer. The Food and Drug Association (FDA) approved the recombinant HPV 9-valent vaccine (GARDASIL 9) for all boys, men, girls, and women between the ages of 9 and 45 years (https://www.fda.gov/vaccinesblood-biologics/vaccines/gardasil-9). Even though most women have been exposed to HPV by the recommended vaccination age of 9 to 26, societies still endorse this recommendation [12••].In older patients between 27 and 45 years, the ACIP recommends individualizing these scenarios and encourages shared clinical decision-making between the patient and the provider based on their risk for acquisition of HPV $[38 \bullet \bullet, 39]$. COVID-19 vaccine

Currently, there are several COVID-19 vaccines that have been used in patients with IBD including mRNA vaccines, viral vector vaccines, and inactivated vaccines. In the USA, the mRNA vaccines, Pfizer BioNTech and Moderna, and the viral vector vaccine from Johnson and Johnson have been approved and can be administered to all patients with IBD. Currently, all patients with IBD should be vaccinated against severe acute respiratory syndrome coronavirus 2 (SARS-CoV-2) at the earliest opportunity to do so and regardless of which vaccine they are offered $[12 \bullet \bullet, 40]$.

Live vaccines

Administration of live vaccines is not recommended for patients on immunosuppressive therapies. Certain immunosuppressed patients who are not severely immunosuppressed may safely receive selected live vaccines; however, this option needs to be considered on a case-by-case basis until more prospective data is available [41]. The common live vaccines include the varicella vaccine, the measles, mumps, and rubella (MMR) vaccine, and the live influenza vaccine. Several travel vaccines are live vaccines, and these include the cholera vaccine, yellow fever vaccine, and one of the available typhoid vaccines.

\section{Considerations for patients on immunosuppression or planned to start immunosuppression}

The IDSA advises that live vaccines should be given at least 4 weeks prior to initiation of immunosuppression and definitely not within 2 weeks. They also advise inactivated vaccines to be given at least 2 weeks prior to initiation of immunosuppression [18]. As for patients who are already receiving immunosuppression, current recommendations are that they should avoid receiving live vaccines. If live vaccines are needed, then patients should be categorized into those with severe immunosuppression and those without significant immunologic compromise. Patients without significant 
immunologic compromise can consider receipt of essential live vaccines on a case-by-case basis after discussion with infectious disease experts. Patients who are considered highly or severely immunosuppressed include those on high dose steroids $\geq 20 \mathrm{mg}$ per day of oral prednisone for $\geq 14$ days, immunomodulator use (azathioprine $\geq 3 \mathrm{mg} / \mathrm{kg} / \mathrm{day}$, 6-mercaptopurine $\geq$ $1.5 \mathrm{mg} / \mathrm{kg} /$ day, or $\geq 0.4 \mathrm{mg} / \mathrm{kg} /$ week), use of anti-TNF agents, ustekinumab, or transplant-related immunosuppressive medications (cyclosporine, tacrolimus, mycophenolate) $[20,35$

Prevalence of osteoporosis in patients with IBD is reported to be as high as $42 \%$, with higher rates among CD patients when compared to UC patients [42-44]. Even in newly diagnosed patients with IBD, and before any medications are started, osteoporosis prevalence is as high as 5\% [45]. The reasons behind low bone mass are related to the inflammatory disease itself, medications that are used particularly corticosteroids, malabsorption especially of vitamin $\mathrm{D}$, as well as dietary restrictions. In a conditional recommendation with very low-level evidence, the 2017 American College of Gastroenterology made a statement that all patients with IBD with conventional risk factors for abnormal bone mineral density should undergo screening for osteoporosis with bone mineral density testing at the time of diagnosis and periodically thereafter $[8 \bullet \bullet$.This recommendation includes patients with a pre-existing fragility fracture, women 65 years and older, men 70 years and older, and those with risk factors for low bone mass including those who have received steroid treatments, who suffered from systemic chronic inflammation, and malnutrition $[45,46]$. Previously, it was thought that having IBD alone is an independent predictor for developing the bone disease, but newer data failed to support this theory. Based on the Crohn's and Colitis Foundation checklist, a DXA scan of the hip and spine should be performed for all high-risk patients with IBD to screen for osteoporosis [11•]. These high-risk patients include patients with a low body mass index (BMI), those with a cumulative steroid exposure longer than 3 months, smokers, post-menopausal women, and those with hypogonadism [11•]. In case the DXA scan is normal, it is recommended to repeat the scan after 5 years [11 $\bullet$ Other experts (Cornerstones Health) recommend serial monitoring of vitamin D levels, and if deficient, appropriate replacement is needed [10•]. As for assessment for osteoporosis, a DXA scan is recommended for any patient with IBD who also has one of the following risk factors: chronic steroid use greater than 3 months, maternal history of osteoporosis, malnourished or very thin (i.e., low BMI), amenorrheic, or if post-menopausal [10•]. Additionally, it is recommended that with any course of steroids, patients also get a prescription of calcium and vitamin $\mathrm{D}[10 \bullet]$. 


\section{Malignancy}

\section{Cervical cancer screening}

There has been the consensus among the European Crohn's and Colitis Organization, the American College of Obstetrics and Gynecology along with the CDC, and the most recent ACG Preventive guidelines that all women with IBD who are receiving systemic immunosuppressive therapy should have an annual cervical cancer screening examination/PAP smear $[8 \bullet \bullet, 47,48]$, (https://www.cdc.gov/std/tg2015/specialpops.htm.)] Up to 70\% of cervical cancer is caused by HPV 16 or 18 infection [37]. Vaccination recommendations are detailed above.

\section{Colon cancer screening}

Patients with more than 8-year history of ulcerative colitis (excluding ulcerative proctitis) and Crohn's colitis involving more than a third of their colon are at an increased risk for colon cancer. Surveillance for colon cancer should begin within 1-2 years after an initial screening colonoscopy, and if negative, surveillance colonoscopies every 1 to 3 years are recommended [49, 50]. Patients with a concomitant history of primary sclerosing cholangitis (PSC) should undergo annual colonoscopy from the time of their PSC diagnosis due to their pronounced risk of colon cancer [51].

Skin cancer screening

Patients with IBD, regardless of immunosuppression, are at an increased risk for the development of skin cancers, both melanoma and nonmelanoma skin cancers (NMSC) [52, 53]. Thiopurines increase the risk of NMSC even further by around 6-fold and this risk persists even after discontinuation of the thiopurine $[8 \bullet \bullet$.Anti-TNF biologic agents, increase the risk of melanoma 2 -fold [54]. For this reason, patients with IBD should be referred to dermatology for a screening exam and determination of the frequency of exams, especially if they are receiving immunosuppressive medications or have had prior use of a thiopurine. Additionally, sunscreen protection is recommended for all patients with IBD, particularly those who will be starting immunosuppressive therapy, to reduce the additional risk.

All patients with IBD, regardless of their disease subtype, should be encouraged to stop smoking due to the negative effect of tobacco smoking on their general well-being and the increased risk for many malignancies including 
lung and colon cancer. Data had shown that smoking may have a favorable protective effect on the development of ulcerative colitis [55]. Crohn's disease on the other hand is negatively affected by tobacco smoking. Not only does smoking increase the risk for the development of Crohn's disease, but smokers tend to develop more complications including strictures as well as the need for repeat surgical resections. Additionally, smoking tobacco has been identified as the only modifiable risk factor for post-operative recurrence of Crohn's disease [56, 57]. Regarding extra-intestinal manifestations of IBD, there have been reports showing increased prevalence of joint and skin manifestations in those patients with tobacco use regardless of the IBD subtype [58].

\section{Mental health screening/depression screening}

Like patients with other chronic illnesses, anxiety and depression are prevalent among patients with IBD compared to the general population. It is critical to screen patients with IBD for anxiety and depression especially since depressive severity was found to be a risk factor for suicidal ideation among these patients, and aggressive and early management can prevent progression to completed suicides [59]. Screening questionnaires for anxiety and depression are readily available and easy to administer. Follow-up for patients with high depression/anxiety scores should be offered and timely referral to their primary care physician or psychiatrist is crucial in order to improve outcomes of these patients [60].

\section{Conclusions}

Staying up-to-date with health care maintenance of patients with IBD is critical, especially given the increased risk for vaccine-preventable infections as well as comorbidities such as malignancies, bone, and mood disorders. There are many checklists developed to inform clinical practice for both gastroenterologists and primary care physicians $[10 \bullet 11 \bullet]$, who should be working hand in hand to provide the best state of the art care for patients with IBD.

\section{Author Contribution}

J.G.H.: drafting of the manuscript; M.F.P.: critical review of the manuscript; F.A.F.: drafting and critical review of the manuscript.

\section{Declarations}

\section{Conflict of Interest}


Jana G. Hashash declares no competing interests. Michael F. Picco declares no competing interests. Dr. Farraye is a consultant for Arena, BMS, Braintree labs, GI Reviewers, GSK, Innovation Pharmaceuticals, Iterative scopes, Janssen, Pfizer and Sebela. He sits on a data safety monitoring board for Lilly and TheraVance.

\section{References and Recommended Reading}

Papers of particular interest, published recently, have been highlighted as:

- Of importance

•- Of major importance

1. Bernstein $\mathrm{CN}$, Blanchard JF, Rawsthorne P, Yu N. The prevalence of extraintestinal diseases in inflammatory bowel disease: a population-based study. Am J Gastroenterol. 2001;96(4):1116-22.

2. Mir FA, Kane SV. Health maintenance in inflammatory bowel disease. Curr Gastroenterol Rep. 2018;20(5):1-6.

3. Selby L, Kane S, Wilson J, et al. Receipt of preventive health services by IBD patients is significantly lower than by primary care patients. Inflamm Bowel Dis. 2008;14(2):253-8.

4. Malhi G, Rumman A, Thanabalan R, et al. Vaccination in inflammatory bowel disease patients: attitudes, knowledge, and uptake. J Crohn's Colitis. 2015;9(6):439-44.

5. Wasan SK, Coukos JA, Farraye FA. Vaccinating the inflammatory bowel disease patient: deficiencies in gastroenterologists knowledge. Inflamm Bowel Dis. 2011;17(12):2536-40.

6. Desalermos AP, Farraye FA, Wasan SK. Vaccinating the inflammatory bowel disease patient. Expert Rev Gastroenterol Hepatol. 2015;9(1):91-102.

7. Melmed GY. Immunizations and IBD: whose responsibility is it? If I'm the prescribing doctor, shouldn't it be mine? Inflamm Bowel Dis. 2012;18(1):41-2. https://doi.org/10.1002/ibd.21666.

8.• Farraye FA, Melmed GY, Lichtenstein GR, Kane SV. ACG clinical guideline: preventive care in inflammatory bowel disease. ACG. 2017;112(2):241-258.

This is a clinical guideline on preventive care for patients with inflammatory bowel disease. This comprehensive review covers vaccinations as well as other health maintenance issues including screening for cervical cancer, depression and anxiety, skin cancers, and osteoporosis.

9. Weaver KN, Long MD. Preventive medicine in inflammatory bowel disease. Clin Gastroenterol Hepatol. 2019;17(5):824-8.
10. https://www.cornerstoneshealth.org/wp-content/ uploads/2020/08/NEW-IBD-Checklist-for-Monit oring-Prevention-526a.pdf.

This is a very useful checklist which may aid gastroenterologists when they evaluate patients with inflammatory bowel disease. The checklist covers vaccine preventable illnesses, bone health, therapy related testing, cancer prevention, amongst other assessments.

11. https://www.crohnscolitisfoundation.org/sites/defau lt/files/2019-09/Health\%20Maintenance\%20Che cklist $\% 202019-3$.pdf.

This is a very useful checklist that was developed by the Crohn's and Colitis Foundation Professional Education Sub-Committee that covers vaccines, infections of concern, cancer screening, and other health maintenance issues such as osteoporosis, depression/anxiety and smoking that must be addressed with all patients with inflammatory bowel disease.

12.• Kucharzik T, Ellul P, Greuter T, et al. ECCO guidelines on the prevention, diagnosis, and management of infections in inflammatory bowel disease. J. Crohn's Colitis. 2021;15(6):879-913. https://doi. org/10.1093/ecco-jcc/jjab052.

This is the most recently published 2021 European Crohn's and Colitis Organisation Guideline on the prevention, diagnosis and management of infections in inflammatory bowel disesases.

13. Rahier J-F, Papay P, Salleron J, et al. H1N1 vaccines in a large observational cohort of patients with inflammatory bowel disease treated with immunomodulators and biological therapy. Gut. 2011;60(4):456-62.

14. Dotan I, Werner L, Vigodman S, et al. Normal response to vaccines in inflammatory bowel disease patients treated with thiopurines. Inflamm Bowel Dis. $2012 ; 18(2): 261-8$.

15. Reich J, Wasan S, Farraye FA. Vaccinating patients with inflammatory bowel disease. Gastroenterol Hepatol. 2016;12(9):540. 
16. Long MD, Gulati A, Wohl D, Herfarth H. Immunizations in pediatric and adult patients with inflammatory bowel disease: a practical case-based approach. Inflamm Bowel Dis. 2015;21(8):1993-2003.

17. Kim DK, Bridges CB, Harriman KH. Advisory committee on immunization practices recommended immunization schedule for adults aged 19 years or older: United States, 2016. Ann Intern Med. 2016;164(3):184-94.

18. Rubin LG, Levin MJ, Ljungman P, et al. 2013 IDSA clinical practice guideline for vaccination of the immunocompromised host. Clin Infect Dis. 2014;58(3):e44-100.

19. Freedman MS, Ault K, Bernstein H. Advisory committee on immunization practices recommended immunization schedule for adults aged 19 years or older-United States, 2021. Morb Mortal Wkly Rep. 2021;70(6):193.

20. https://wwwnc.cdc.gov/travel/yellowbook/2020/ travelers-with-additional-considerations/immun ocompromised-travelers. Accessed May 23.

21. Marín AC, Gisbert JP, Chaparro M. Immunogenicity and mechanisms impairing the response to vaccines in inflammatory bowel disease. WJG. 2015;21(40):11273.

22. Visser L. The immunosuppressed traveler. Infect Dis Clin. 2012;26(3):609-24.

23. Tinsley A, Navabi S, Williams ED, et al. Increased risk of influenza and influenza-related complications among 140,480 patients with inflammatory bowel disease. Inflamm Bowel Dis. 2019;25(2):369-76.

24. Stobaugh DJ, Deepak P, Ehrenpreis ED. Hospitalizations for vaccine preventable pneumonias in patients with inflammatory bowel disease: a 6-year analysis of the Nationwide Inpatient Sample. Clin Exp Gastroenterol. 2013;6:43.

25. Andrisani G, Frasca D, Romero $M$, et al. Immune response to influenza $\mathrm{A} / \mathrm{H} 1 \mathrm{~N} 1$ vaccine in inflammatory bowel disease patients treated with anti TNF-a agents: effects of combined therapy with immunosuppressants. J Crohn's Colitis. 2013;7(4):301-7.

26. Hagihara Y, Ohfuji S, Watanabe $\mathrm{K}$, et al. Infliximab and/or immunomodulators inhibit immune responses to trivalent influenza vaccination in adults with inflammatory bowel disease. J Crohn's Colitis. 2014;8(3):223-33.

27. Melmed GY. Vaccinations while on thiopurines: some protection is better than none. Am J Gastroenterol. 2012;107(1):141.

28. Launay O, Abitbol V, Krivine A, et al. Immunogenicity and safety of influenza vaccine in inflammatory bowel disease patients treated or not with immunomodulators and/or biologics: a two-year prospective study. J Crohn's Colitis. 2015;9(12):1096-107.

29. deBruyn J, Fonseca K, Ghosh S, et al. Immunogenicity of influenza vaccine for patients with inflammatory bowel disease on maintenance infliximab therapy: a randomized trial. Inflamm Bowel Dis. 2016;22(3):638-47.

30. Caldera F, Hayney MS, Farraye FA. Vaccination in patients with inflammatory bowel disease. ACG. 2020;115(9):1356-1361.

31. Caldera F, Hillman L, Saha S, et al. Immunogenicity of high dose influenza vaccine for patients with inflammatory bowel disease on anti-TNF monotherapy: a randomized clinical trial. Inflamm Bowel Dis. 2020;26(4):593-602.

32. Long MD, Martin C, Sandler RS, Kappelman MD. Increased risk of pneumonia among patients with inflammatory bowel disease. Am J Gastroenterol. 2013;108(2):240.

33. Kantsø B, Simonsen J, Hoffmann S, ValentinerBranth P, Petersen AM, Jess T. Inflammatory bowel disease patients are at increased risk of invasive pneumococcal disease: a nationwide Danish cohort study 1977-2013. Am J Gastroenterol. 2015;110(11):1582-7.

34. Van Aalst M, Garcia Garrido HM, Van Der Leun $\mathrm{J}$, et al. Immunogenicity of the currently recommended pneumococcal vaccination schedule in patients with inflammatory bowel disease. Clin Infect Dis. 2020;70(4):595-604.

35. Caldera F, Hayney MS, Cross RK. Using number needed to harm to put the risk of herpes zoster from tofacitinib in perspective. In: Oxford University Press US. 2019;25(6):955-7. https://doi.org/10.1093/ibd/ izy387.

36. Khan N, Patel D, Trivedi C, et al. Overall and comparative risk of herpes zoster with pharmacotherapy for inflammatory bowel diseases: a nationwide cohort study. Clin Gastroenterol Hepatol. 2018;16(12):1919-1927. e1913.

37. Kane S, Khatibi B, Reddy D. Higher incidence of abnormal Pap smears in women with inflammatory bowel disease. Am J Gastroenterol. 2008;103(3):631-6.

$38 . \bullet$ Freedman MS, Bernstein H, Ault KA. Recommended Adult Immunization Schedule, United States, 2021. Annals of Internal Medicine. 2021; 174(3):374-384.

This document summarizes the recommended immunization schedule for adult patients in the United States.

39. Meites E, Szilagyi PG, Chesson HW, Unger ER, Romero JR, Markowitz LE. Human papillomavirus vaccination for adults: updated recommendations of the Advisory Committee on Immunization Practices. In: Wiley Online Library; 2019.

40. Siegel CA, Melmed GY, McGovern DP, et al. SAR$S C o V-2$ vaccination for patients with inflammatory bowel diseases: recommendations from an international consensus meeting. Gut. 2021;70(4):635-40.

41. Croce E, Hatz C, Jonker EF, Visser L, Jaeger VK, Bühler S. Safety of live vaccinations on immunosuppressive therapy in patients with immune-mediated inflammatory diseases, solid organ transplantation or after bone-marrow transplantation-a systematic 
review of randomized trials, observational studies and case reports. Vaccine. 2017;35(9):1216-26.

42. Lichtenstein GR, Sands BE, Pazianas M. Prevention and treatment of osteoporosis in inflammatory bowel disease. Inflamm Bowel Dis. 2006;12(8):797-813.

43. Hashash JG, Binion DG. Exercise and inflammatory bowel disease: insights into etiopathogenesis and modification of clinical course. Gastroenterol Clin. 2017;46(4):895-905.

44. Ali T, Lam D, Bronze MS, Humphrey MB. Osteoporosis in inflammatory bowel disease. Am J Med. 2009; 122(7):599-604.

45. Bernstein CN, Leslie WD, Leboff MS. AGA technical review on osteoporosis in gastrointestinal diseases. Gastroenterology. 2003;124(3):795-841.

46. Vestergaard P. Bone loss associated with gastrointestinal disease: prevalence and pathogenesis. Eur J Gastroenterol Hepatol. 2003;15(8):851-6.

47. Magro F, Peyrin-Biroulet L, Sokol H, et al. Extraintestinal malignancies in inflammatory bowel disease: results of the 3rd ECCO Pathogenesis Scientific Workshop (III). J Crohn's Colitis. 2014;8(1):31-44.

48. Obstetricians ACo, Gynecologists. Practice bulletin no. 157: cervical cancer screening and prevention. Obstet Gynecol. 2016;127(1):e1-e20.

49. Lichtenstein GR, Loftus EV, Isaacs KL, Regueiro MD, Gerson LB, Sands BE. ACG clinical guideline: management of Crohn's disease in adults. Official journal of the American College of Gastroenterology| ACG. 2018;113(4):481-517.

50. Long MD, Sands BE. When do you start and when do you stop screening for colon cancer in inflammatory bowel disease? Clin Gastroenterol Hepatol. 2018;16(5):621-3.

51. Ekbom A, Helmick C, Zack M, Adami H-O. Ulcerative colitis and colorectal cancer: a population-based study. N Engl J Med. 1990;323(18):1228-33.

52. Singh H, Nugent $Z$, Demers AA, Bernstein CN. Increased risk of nonmelanoma skin cancers among individuals with inflammatory bowel disease. Gastroenterology. 2011;141(5):1612-20.

53. Singh S, Nagpal SJS, Murad MH, et al. Inflammatory bowel disease is associated with an increased risk of melanoma: a systematic review and meta-analysis.

Clin Gastroenterol Hepatol. 2014;12(2):210-8.

54. Moran G, Lim A, Bailey J, et al. dermatological complications of immunosuppressive and anti-TNF therapy in inflammatory bowel disease. Aliment Pharmacol Ther. 2013;38(9):1002-24.

55. Vedamurthy A, Ananthakrishnan AN. Influence of environmental factors in the development and outcomes of inflammatory bowel disease. Gastroenterol Hepatol. 2019;15(2):72.

56. Hashash JG, Regueiro M. A practical approach to preventing postoperative recurrence in Crohn's disease. Curr Gastroenterol Rep. 2016;18(5):25.

57. Hashash JG, Regueiro MD. The evolving management of postoperative Crohn's disease. Expert Rev Gastroenterol Hepatol. 2012;6(5):637-48.

58. Severs M, van Erp SJ, Van Der Valk M, et al. Smoking is associated with extra-intestinal manifestations in inflammatory bowel disease. J Crohn's Colitis. 2016;10(4):455-61.

59. Hashash JG, Vachon A, Ramos Rivers C, et al. Predictors of suicidal ideation among IBD outpatients. J Clin Gastroenterol. 2019;53(1):e41-5.

60. Deter H-C, Keller W, von Wietersheim J, Jantschek G, Duchmann R, Zeitz M. Psychological treatment may reduce the need for healthcare in patients with Crohn's disease. Inflamm Bowel Dis. 2007;13(6):745-52.

\section{Publisher's Note}

Springer Nature remains neutral with regard to jurisdictional claims in published maps and institutional affiliations. 